Results Experts recorded (con)structural upgrades in the course of the generation of a school route map and claimed the maps to be of help in road safety education. $65 \%$ of the questioned parents indicated that they got the map at start of school, although every year one pupil should get one, and only $10 \%$ really used it. To improve the usage they recommended handing out the plan already in Kindergarten. In contrast, about $70 \%$ of the headmasters of schools with school route maps stated that the map is used in road safety education. They saw the major problem in the annual reprint. Of the schools without a map $86 \%$ didn't know such maps are existing and about 50\% declared that they would be interested. The accident analysis revealed no significant changes in the accident rate as well as in the casualties since the introduction of school route maps.

Conclusions Regarding the accident rate no success of Austrian school route maps could be proven. However, experts and headmasters stated positive experiences. Overall seemed to be room for improvement. One of the first steps would be to make school route maps more known, and to hand them out to parents of year one pupils earlier and yearly (e.g. on school registration day).

\section{PERCEPTION OF UNINTENTIONAL CHILDHOOD INJURIES AMONG RURAL MOTHERS IN SOUTH INDIA}

'Leeberk Raja Inbaraj, ${ }^{2}$ Anuradha Rose, ${ }^{2}$ Kuryan George, ${ }^{2}$ Anuradha Bose. ${ }^{1}$ Bangalor Baptist Hospital, Bangalore, India; ${ }^{2}$ Christian Medical College, Vellore, India

\subsection{6/injuryprev-2016-042156.651}

Background Parental perception of safe and risk-free environment is critical in the prevention of unintentional childhood injury. An accurate perception of risky situations is essential to minimise the rate of childhood injuries. Knowledge on parental perception in rural India will be helpful in planning preventive strategies. This study was conducted to assess parental perception on risks and hazards leading to unintentional childhood injuries.

Methods A cross sectional study was conducted in 13 clusters of a rural block in South India. Double stage cluster sampling method was used to recruit 100 mothers and they were interviewed using a tool developed by Glik and Kronenfeld. Two FGDs were also conducted.

Results Mothers' perception of likelihood of injury from hazards such as household door and drawers, small toys, plastic bags and cribs was poor. These objects were also perceived as less dangerous hazards. Mothers had a poor perception of injury by entrapment in refrigerators, choking and strangulation by a rope or a cord. Choking bruises, puncture wounds were perceived as less serious events. Age, education and literacy were found to be significant predictors of perception of risk and hazard in univariate analysis, the logistic regression model did not show any significant associated factors for perception of risk and hazard. $9 \%$ of mothers believed injuries can be completely prevented and illiteracy $(\mathrm{p}=0.04)$ was associated with poor perception on prevention in univariate analysis. Few mothers in FGD believed that injuries cannot be prevented as it is natural (Vidhi) for children to sustain injuries.

Conclusions It is a fact that the mother's literacy and schooling is closely related to child health and survival. Promotion of injury preventive engineering strategies and enforcement will only be effective when the literacy of the mother increases. Health education can improve maternal perception and have a positive impact on prevention of unintentional injuries.

\section{STRENGTHENING A CULTURE OF SAFETY FOR CHILDREN: DEVELOPING HOSPITAL AND COMMUNITY COLLABORATIONS}

Nan Peterson, James Savage, Nicole Vesely, Rishelle Eithun. UW Health American Family Children's Hospital, USA

\subsection{6/injuryprev-2016-042156.652}

Background Unintentional injury is the leading cause of death and disability for America's children. The economic consequences of injury are staggering; with injury being the leading cause of medical spending for children ages $5-14$ in Wisconsin. As a health care system, we see the consequences of preventable injuries. As a children's hospital we have an obligation to lead the way in modelling best practice, evidence-based injury prevention strategies for children in collaboration with our community partners.

Methods/approach Review of Wisconsin paediatric injury and death data formed the basis of prioritising program development or system-level strategies for injury prevention. Using a policy, systems, environmental approach, we identified best practice injury prevention strategies with our community partners. By implementing a collective impact model and community engagement, we formulated plans for improving the injury prevention strategies for children and families in our community.

Results Examples related to child passenger safety, home visitor program, a safe sleep campaign, Safety Town, and a "safety store" will be provided. Program barriers and challenges, as well as successful outcomes in strengthening the culture of injury prevention through community engagement will be shared.

Conclusions Health care providers and community partners are looking to embrace population health strategies to achieve a greater good for improving the health of children. Using a collective impact model enables communities to accelerate the progress they can have in reducing childhood injury related morbidity and mortality. Safety devices, when correctly used, are highly effective in preventing injuries and saving lives. Recognition of the need to reduce health disparities by removing potential social, economic and language barriers for families around injury prevention strategies is critical.

\section{SAFETY FOR YOUTH INVOLVED IN COMMUNITY BASED AGRICULTURE}

Marsha Salzwedel, Bryan Weichelt, Casper Bendixsen, Barbara C Lee. National Children's Centre for Rural and Agricultural Health and Safety, Marshfield, WI, USA

\subsection{6/injuryprev-2016-042156.653}

Background As Community Based Agriculture (CBA) gains popularity in the U.S., more youth are becoming involved. One type of CBA is Community Supported Agriculture (CSA). In CSA, farms sell shares of their harvest, which may include customer labour: as part of the share, to reduce the price of the share, or to gain extra product. Another form of CBA is public gardens (e.g., community, urban, school). Adults work with youth to plant, weed and harvest. Often, those working in CBA are not familiar with the tasks, equipment, and hazards. There are no known safety resources for youth involved in CBA, thus adults depend on prevention strategies from various disciplines to address common problems such as bee stings or weather-related hazards.

Methods A pilot study was undertaken to assess youth safety in CBA. A survey was sent to a convenience sample of community/ 
school garden organisers to learn more about youth working in gardens, the types of jobs they perform, the types of equipment being used, and the injuries and illnesses that occur. Eighteen completed surveys were returned and analysed, representing a variety of operations.

Results Survey results revealed many groups engage youth in CBA, and youth perform a wide variety of tasks. Very few have formal safety programs; all desire more safety information. In response, "Community-Based Agriculture: Safety Guidelines for Youth Working in Gardens" was developed. Content is based on the North American Guidelines for Children's Agricultural Tasks and Safety Guidelines for Hired Adolescent Farm Workers. Dissemination efforts included press releases, Ag Educator listservs, and social media. Over 1000 booklets have been distributed, and links are on several national websites.

Conclusions In the U.S., CBA activities offer an opportunity for safety professionals to engage with community programs and embed effective principles to safeguard youth. This is especially important, given the national push to grow and eat natural foods.

\section{HOW DO MOTHERS ENGAGE WITH NEWS COVERAGE OF PAEDIATRIC INJURY RESEARCH? AN EXPLORATORY, MIXED-METHODS STUDY}

\begin{abstract}
${ }^{1,2}$ Katherine C Smith, ${ }^{3}$ Jennifer A Manganello, ${ }^{4}$ Kristin Roberts, ${ }^{4}$ Roxanne Kaercher, 4,5,6 Lara McKenzie. 'Department of Health, Behaviour and Society, Johns Hopkins Bloomberg School of Public Health, Baltimore, MD; ${ }^{2}$ Centre for Injury Research and Policy, Johns Hopkins Bloomberg School of Public Health, Baltimore, MD; ${ }^{3}$ Department of Health Policy and Management, University at Albany School of Public Health, State University New York, Rensselaer, NY; ${ }^{4}$ Centre for Injury Research and Policy, Research Institute at Nationwide Children's Hospital, Columbus, $\mathrm{OH}_{;}^{5}$ Department of Paediatrics, the Ohio State University College of Medicine, Columbus, $\mathrm{OH}_{;}{ }^{6}$ Division of Epidemiology, the Ohio State University College of Public Health, Columbus, $\mathrm{OH}$
\end{abstract}

\subsection{6/injuryprev-2016-042156.654}

Objective To examine understanding of and engagement with news messages about paediatric injury research findings in mothers of young children.

Methods We conducted six focus groups with 49 mothers of young children $(<6$ years of age) in which participants were shown three videos of televised news stories discussing paediatric injury research (car seats, household poisoning, high chairs). After viewing each story, mothers individually completed recall exercises and questions about engagement with story elements and then discussed reactions to injury content.

Results Almost all mothers who participated (94-98\%) recalled key information about the injury event depicted, but recall of statistical information was not as robust (51-82\%). Prevention guidelines were 'most interesting' to a few mothers (16-18\%); however, the majority was most interested in the narrative/story or the number of injuries presented. Mothers' discussion of story content revealed clear engagement with the dangers posed and the stories presented. Specific elements of the narrative presented in the story shaped engagement as did mothers' understanding of the statistics presented and the way that the research process was understood.

Conclusion Researchers and communication professionals who design news stories intended to convey important injury information should consider whether the story elements (type of family portrayed, use of statistics) will be compelling to the intended audience. Our study suggests that including guidelines and recommendations on how to mitigate the injury hazard are important to mothers of young children, while statistical information needs to be contextualised if presented, as it is not necessarily compelling or easily comprehensible.

\section{NEXT GENERATION OF AGRICULTURAL WORK GUIDELINES FOR YOUTH}

Marsha Salzwedel, Bryan Weichelt, Barbara C Lee, Casper Bendixsen. National Children's Centre for Rural and Agricultural Health and Safety, Marshfield, WI, USA

\subsection{6/injuryprev-2016-042156.655}

Background The North American Guidelines for Children's Agricultural Tasks (NAGCAT) were released in 1999 and Safety Guidelines for Hired Adolescent Farmworkers (SaGHAF) were later released by the National Children's Centre for Rural and Agricultural Health and Safety. Research has demonstrated these guidelines are effective in reducing injuries and now serve as the "gold standard" for assigning agricultural work to youth. Sixteen years after the launch of NAGCAT, there are shortcomings, including inaccurate/incomplete recommendations, new types of jobs, and some guidelines that should be retired. Currently, guidelines are available only in paper format. Today's farm parents prefer to access resources digitally and use mobile devices, so these guidelines need to be updated to fit with today's technology.

Methods An advisory team was formed to assist in gathering knowledge about the type of work youth perform on farms, how they are being injured, and to advise on content. A literature search provided scientific evidence to support advisors' recommendations related to child development and occupational safety. Agricultural safety experts were consulted for content and practicality of updated work guidelines. Technology experts were engaged to develop mobile-responsive materials.

Results This project creates a set of agricultural work guidelines for youth, ages 7-18, that are based upon previously developed and proven guidelines, are technologically injected, digitally accessible, responsive to emerging work methods and hazards, and culturally relevant with language translation options.

Conclusions The NAGCAT/SaGHAF resources are an effective tool in reducing childhood agricultural injuries, but in paper format, the distribution and application is limited. By updating these resources and creating new delivery systems, it increases the likelihood they will continue to be an effective resource for guiding the assignment of jobs to youth working in agriculture.

\section{PAEDIATRIC INJURY FROM MOTORCYCLES AND OFF ROAD VEHICLES}

${ }^{1,2}$ Christopher S Mulligan, ${ }^{2} J u l i e$ Brown. ${ }^{1}$ Prince of Wales Clinical School, University of New South Wales, Australia; ${ }^{2}$ Neuroscience Research Australia, Sydney, Australia

\subsection{6/injuryprev-2016-042156.656}

Background The use of powered off road vehicles including motorcycles, quad-bikes and off-road vehicles, is a leading cause of injury and death in children. To inform injury prevention measures, there is a need to identify risk factors injury related to the vehicle, rider, the riding environment and type of riding being undertaken.

Methods Our aims are to investigate the factors that increase the risk of crashing among children using powered off road vehicles. We are conducting a prospective in-depth case-control study. 